\title{
Research Paper \\ Increasing the Catalytic Power of the Flavin Reductase DszD Enzyme Using Site-Directed Mutagenesis Method in Rhodococcus Erythropolis
}

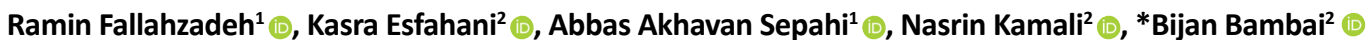

1. Department of Microbiology, Tehran North Branch of Islamic Azad University, Tehran, Iran.

2. Department of Medical Genetics, Institute of Medical Biotechnology, National Institute of Genetic Engineering and Biotechnology (NIGEB), Tehran, Iran.

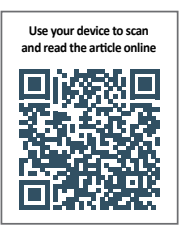

Citation: Fallahzadeh R, Esfahani K, Akhavan Sepahi A, Kamali N, Bambai B. [Increasing the Catalytic Power of the Flavin Reductase DszD Enzyme using Site-Directed Mutagenesis Method in Rhodococcus Erythropolis (Persian)]. Journal of Arak University of Medical Sciences (JAMS). 2019; 22(5):68-77. https://doi.org/10.32598/JAMS.22.5.68

dol'https://doi.org/10.32598/JAMS.22.5.68

Key words:

Flavin reductase DszD enzyme, Bacterial desulfurization, Catalytic power

\section{A B S TRACT}

Article Info:

Background and Aim The flavin reductase DszD enzyme is a key enzyme for providing required reduction potential in the bacterial desulfurization process. Considering the low speed of desulfurization process because of low catalytic power of this enzyme, it is necessary to increase the catalytic power of flavin reductase for industrial use of this enzyme as biocatalyst.

Methods \& Materials The three-dimensional structure of the flavin reductase DszD enzyme was predicted by a CPHmodel server and its amino acid sequence was searched in the protein data bank to identify the homologue molecules. Based on the alignment of the amino acid sequence and the model molecules, the key residues at the flavin mononucleotide substrate were identified. The key residue of asparagine at position 77 was replaced with phenylalanine using the site-directed mutagenesis method.

Ethical Considerations This study with research ethics code IR.NIGEB.EC.1398.6.24 A has been approved by research ethics committee at National Institute of Genetic Engineering and Biotechnology, Tehran, Iran.

Results The cloning and expression of each of the wild-type and mutant genes were performed separately. The catalytic power of the produced wild-type and mutant enzymes were compared. The catalytic activity measurements showed that the mutant enzyme had a 2.5 fold increase in catalytic power. Conclusion Replacing phenylalanine with asparagine at position 77 of flavin reductase DszD enzyme leads to an increase in enzyme catalytic power to increase the speed of bacterial desulfurization process.

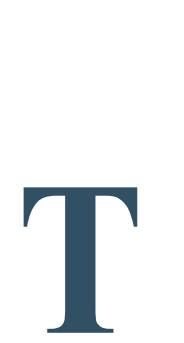

\section{Extended Abstract}

\section{Introduction}

he combustion of petroleum compounds, due to the release of sulfur oxides, can cause numerous environmental issues, like air pollution, as well as many cardiac, respiratory, dermatological, and gastroin- testinal diseases in individuals. Various approaches are applied to reduce the sulfur content of petroleum compounds in the petroleum refinery; chemical desulfurization is the most frequently used method in this respect. The chemical desulfurization is inefficient and requires powerful thermal systems, high pressure, high energy consumption, and costly metal catalysts. The microbial desulfurization method has recently been developed. The microbial desulfurization could remove sulfur in the polycyclic derivatives of petro-

* Corresponding Author:

Bijan Bambai, PhD.

Address: Department of Medical Genetics, Institute of Medical Biotechnology, National Institute of Genetic Engineering and Biotechnology (NIGEB), Tehran, Iran. Tel: +98 (21) 22939413

E-mail: bambai@nigeb.ac.ir 
leum without breaking the carbon skeleton and reducing fuel energy value along the $4 \mathrm{~S}$ route. This pathway was first introduced in the gram-negative Rhodococcus erythropolis bacteria (Rhodococcus erythropolis IGTS8). This bacterium uses sulfur in dibenzothiophene and its derivatives as the sole nutrition source. The active desulfurization reaction is performed in the presence of the flavin reductase DszD enzyme. Naturally, this enzyme has low catalytic power; therefore, it is considered as the primary limiting factor in the desulfurization process rate in the refining industry. The current study aimed to increase the catalytic power of the target enzyme. Thus, the asparagine residue at position 77 was replaced with phenylaniline by targeted mutagenesis. Eventually, the catalytic power of wild and mutated enzymes was compared.

\section{Materials and Methods}

The DszD enzyme amino acid sequence is accessible at the National Center for Biotechnology Information (NCBI) database under the code AAC38226.1. To identify homologous molecules with acceptable similarity, the target enzyme sequence was searched in the protein database. Among the identified homologs, those bounded to the FMN substrate were selected, and their alignment was performed using the Basic Local Alignment Search Tool (BLAST). The wild-type dszD gene was amplified by Polymerase Chain Reaction (PCR) using forward and backward primers with cleavage sites for BamHI and EcoRI restriction enzymes, respectively. The dszD gene was mutated at position 77 using SOEing-PCR. The mutated wild-type gene of $600 \mathrm{bp}$ length was individually cloned into the pET-23a $(+)$ expression vector, then transferred to E. coli BL21 (DE3) cells. Cloning accuracy was assessed using the two restriction enzymes mentioned above and sequencing. Wild and mutated proteins were separately expressed and confirmed by the Western blotting technique. Next, their optical absorption was measured at $340 \mathrm{~nm}$ during the oxidation of NADH to NAD per time unit. Protein concentration was determined by the Bradford method with Bovine Serum Albumin (BSA) as the standard protocol.

\section{Results}

Searching for the amino acid sequence of the flavin reductase DszD enzyme in the protein database has introduced homologous molecules with distinct crystallographic structure. Homologous molecules with the highest structural

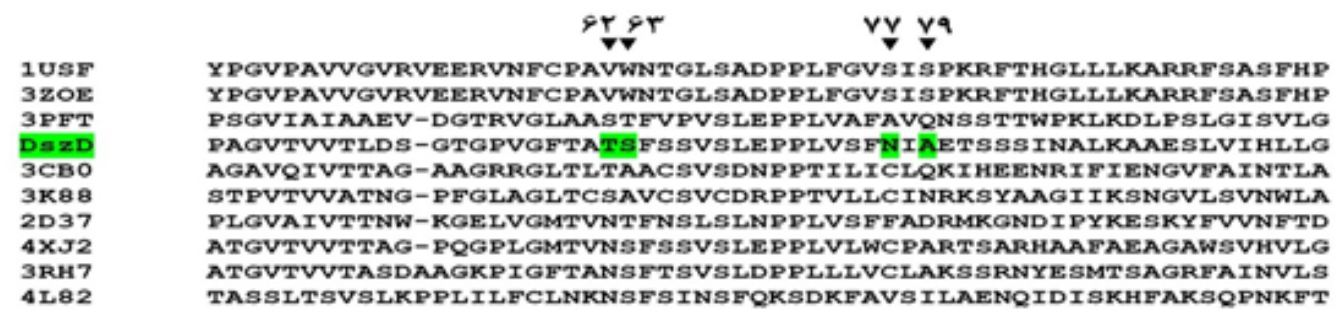

Figure 1. The alignment results of the amino acid sequence of flavin reductase DszD enzyme with homologous enzymes

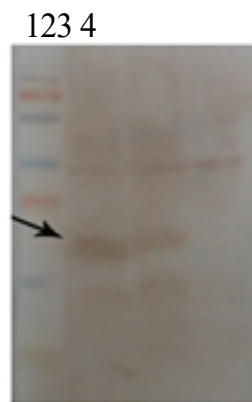

C

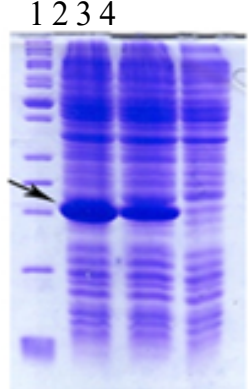

B

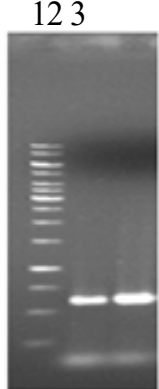

A

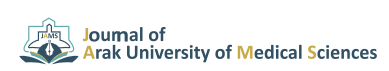

Figure 2. (A) Agarose gel: Column 1: The molecular markers of the type $1 \mathrm{~kg}$ base pair. Column 2: dszD PCR product lacking a 600 bp mutation. Column 3: SOEing-PCR end product with a mutation in alanine 77 and 600 bp length. B. SDS-PAGE gel: Column 1: Protein molecular marker, Column 2: Wild-type recombinant strain, and Column 3: Recombinant mutant strain. Column 4: Strain containing the non-recombinant plasmid; C. the Western blot results of the study samples (B) i.e. visible in columns 2 and 3 of the flavin reductase DszD enzyme in wild and mutated types, respectively. 
similarity were selected as appropriate models for identifying key positions. The alignment of the amino acid sequence of DszD enzyme with the selected homologous molecules revealed that the target enzyme active site includes 4 key positions for binding to the FMN substrate; threonine 62, serine 63, asparagine 77, and alanine 79 (Figure 1).

Wild-type dszD gene amplification was performed applying specific primers (Figure 2a, Column 1). The mutated dszD gene was also obtained at position 77 by a mutant primer (Figure 2a, Column 2). After the enzymatic digestion of the pET-23a(+) vector and the final amplified fragments, each was separately ligated to generate the recombinant plasmid during the binding reaction.

The recombinant plasmids containing the wild- and mutated-type genes were individually transferred to the E.coli BL21 (DE3) expression strain. The experimental results indicated the appropriate expression of the target cloned genes; it had a specific band on the polyacrylamide gel with a molecular weight of $24 \mathrm{kDa}$ (Figure 2b).

Western blotting analysis supported the presence of a significant level of expression of the wild and mutated types of recombinant flavin reductase DszD enzyme (Figure 2c). We compared the mean catalytic power of the wild-type and mutant-type flavin reductase DszD. The obtained data revealed that the mutant enzyme had a catalytic capacity of $392 \pm 6 \mathrm{U} / \mathrm{mg}$, suggesting a 2.5 -fold increase in activity, compared to the wild-type enzyme $(160 \pm 4 \mathrm{U} / \mathrm{mg})$.

\section{Conclusion}

The achieved data suggested that the target enzyme had an appropriate potential to increase the catalytic power in the $4 \mathrm{~S}$ route. Thus, the targeted mutation in the flavin reductase DszD enzyme and its activity in recombinant host cells could increase the desulfurization process efficacy, using the bacterial system. It is suggested that different mutant molecules capable of mutation be produced in several key positions, and their catalytic potency be compared with those reported.

\section{Ethical Considerations}

\section{Compliance with ethical guidelines}

This study with research ethics code IR.NIGEB. EC.1398.6.24 A has been approved by research ethics committee at National Institute of Genetic Engineering and Biotechnology, Tehran, Iran.

\section{Funding}

This research did not receive any specific grant from funding agencies in the public, commercial, or not-forprofit sectors.

\section{Authors' contributions}

Conceptualization, methodology, investigation, writingoriginal draft, writing-review \& editing: All author.

\section{Conflicts of interest}

The authors declared no conflict of interest. 


\title{
افزايش قدرت كاتاليتيكى آنزيم فلاوين ردوكتاز DszD با استفاده از روش جهشز إيى هدفمند در باكترى رودوكوكوس اريترويوليس أنيس فلاوي
}

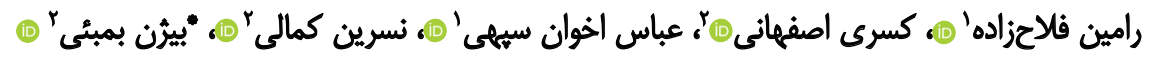 \\ 1. كروه ميكروبيولوري، دانشكده علوم زيستى، واحد تهران شمال، دانشعاه آزاد اسلامى، تهران، يران.

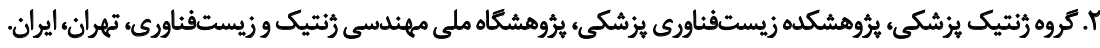

\begin{abstract}
هيكيد

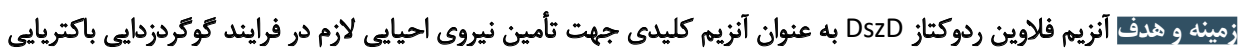

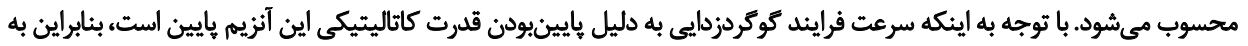

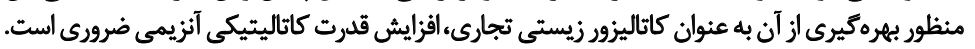

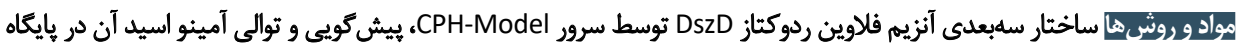

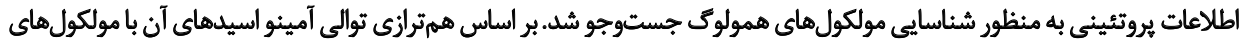

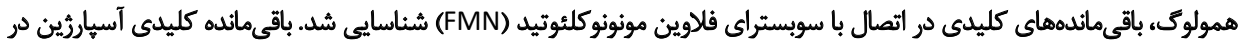

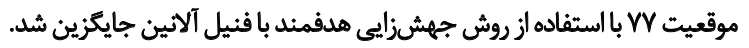

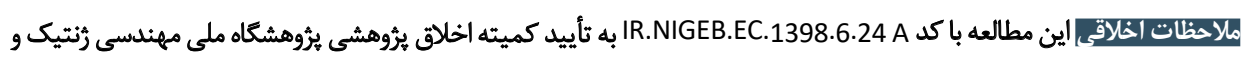
زيستفُناورى رسيده است.

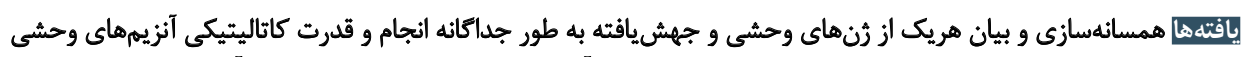

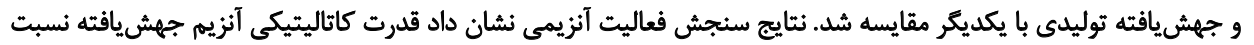

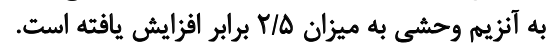

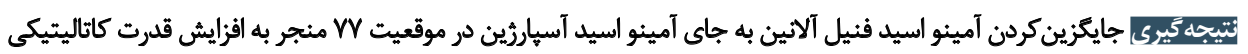

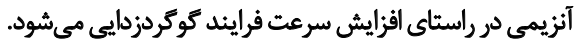

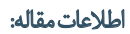

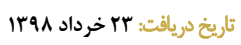

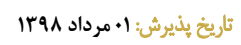

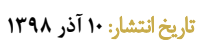

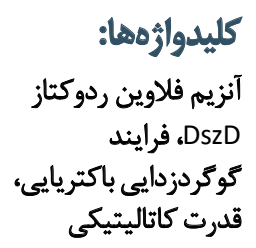

اين فرايند يك واكنش كاتاليتيكى است كه در آن كوكرد

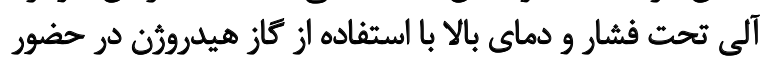

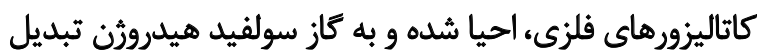

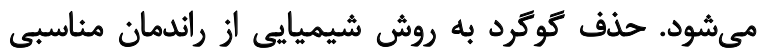

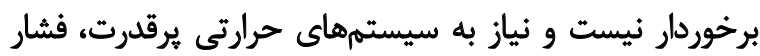

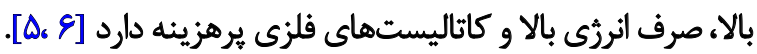

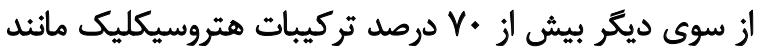

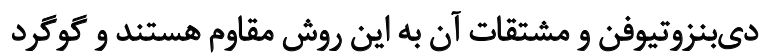

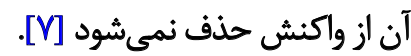

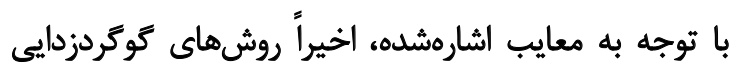

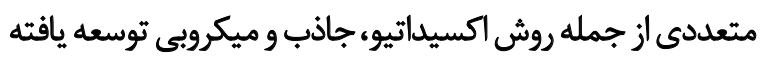

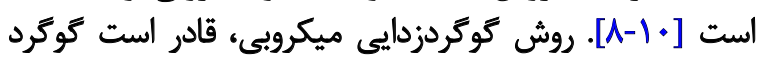

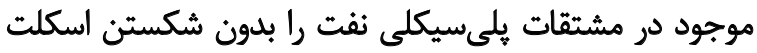

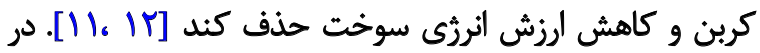

dolês

استفاده از سوختهاي فسيلى و تركيبات نفتي، سبب

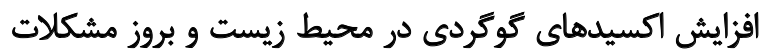

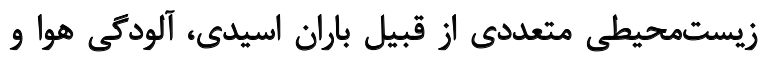

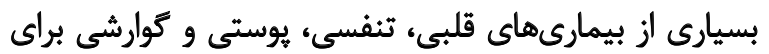

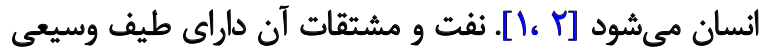

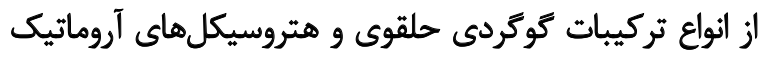

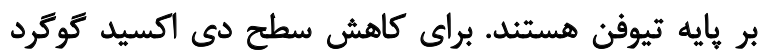

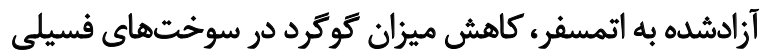

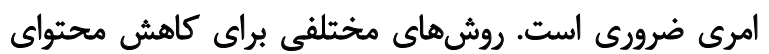

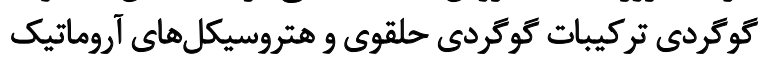

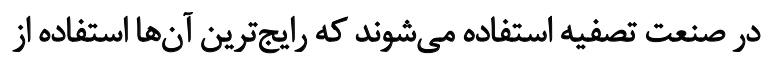

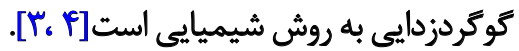

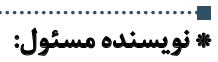

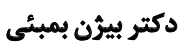

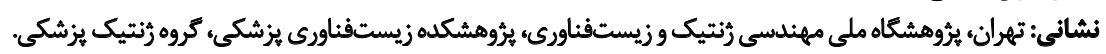

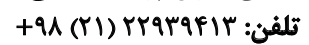


دسترسى AAC38226.1 موجود است. به منظور شناسايى

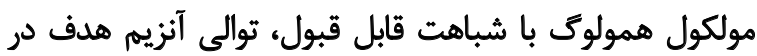

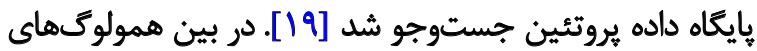

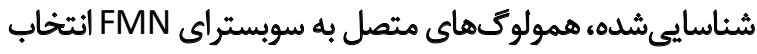
و ههمرازى آنها با يكديكر از طريق ابزار BLAST انجام شد.

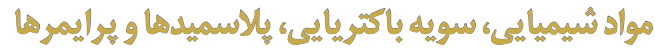

رنومى از سويه باكتريايى رودوكوكوس اريترويوليس DNA

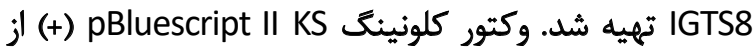

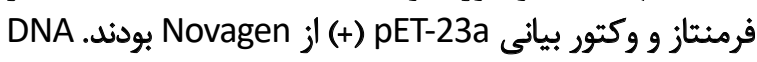

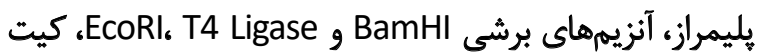

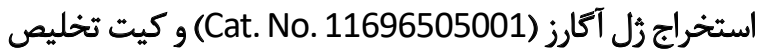

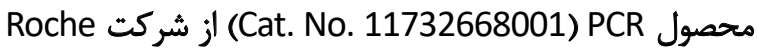

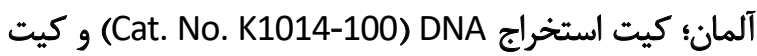

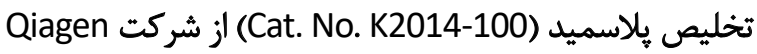
و نشانكرهاي بروتئينى و آنتىبادى T7 tag از شركت فرمنتاز

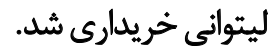

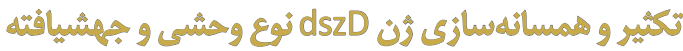

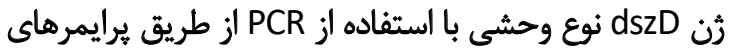
يبشرو و بسرو به ترتيب - ATGGATCCATGTCTGACAAGC CGAATGC , GTAGAATTCCTACTATTGACCTAACGتكثير شد كه به ترتيب داراى جايكاه برش براى

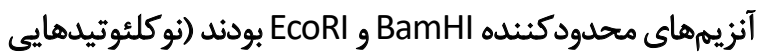

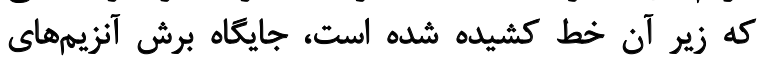

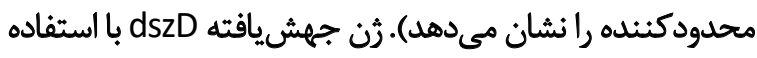

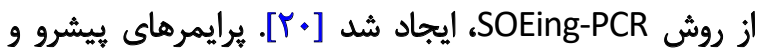

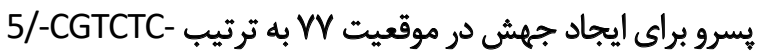

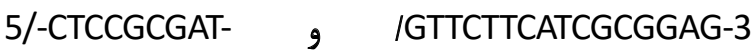

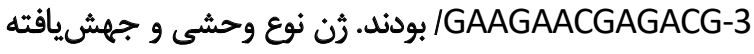

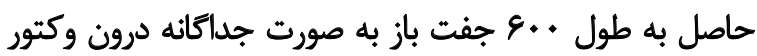

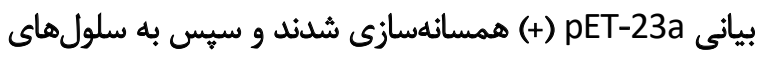

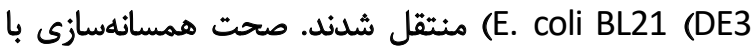

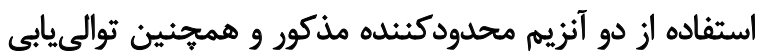

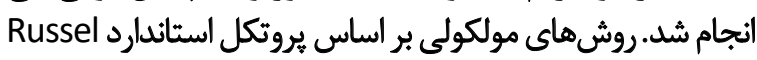
Sambrook ,

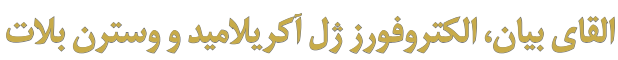

وكتورهاى بيانى

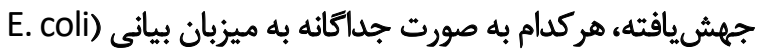

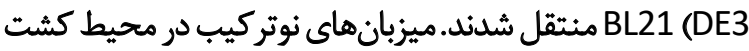

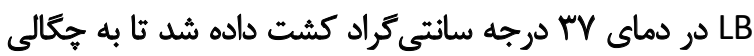

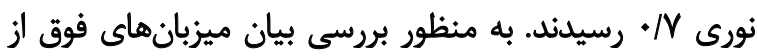

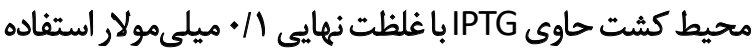

راستاى توسعه اين روش ميكرواركانيسمهاى متعددى شناسايى

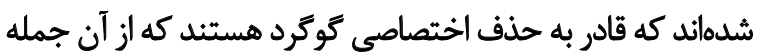

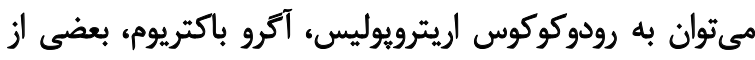

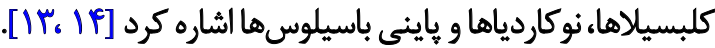

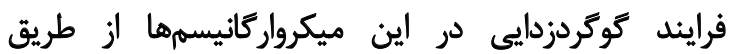

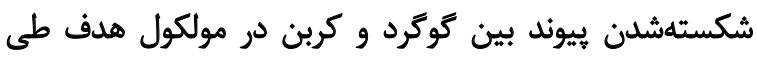

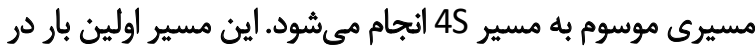

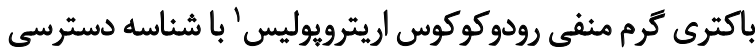

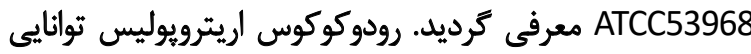

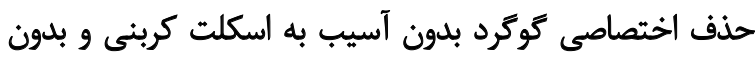

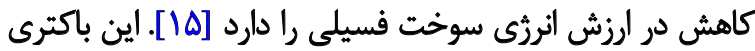

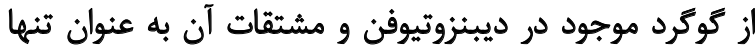

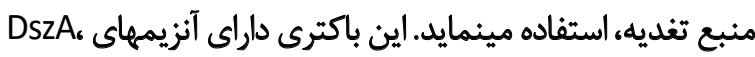
هونت كه آنزيمهاى DszD و DszB، DszC مونواكسيرناز، آنزيم DszB دسولفيناز و آنزيم DszD، يك فلاوين آناوين

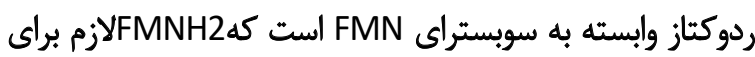

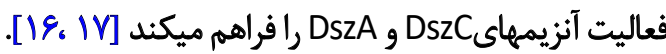

DszD واكنش فعال توكردزدايى در حضور فلاوين ردوكتاز

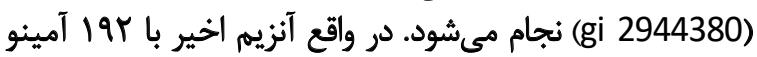

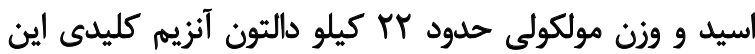

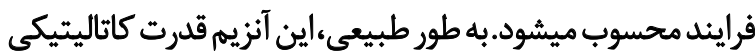

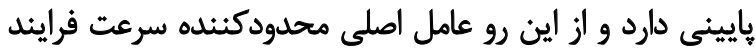

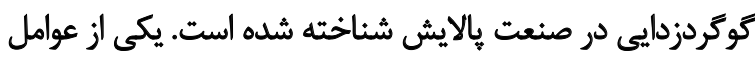

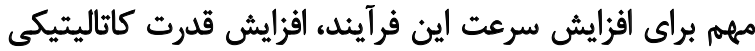

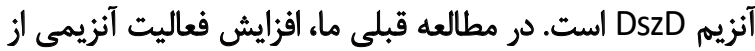

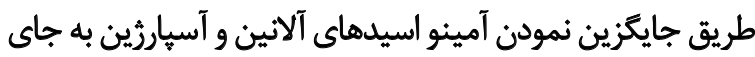

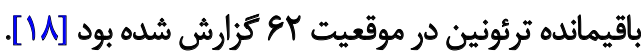

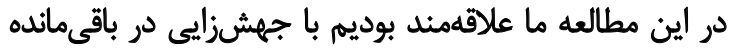

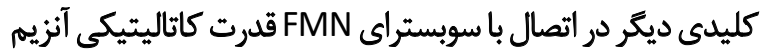

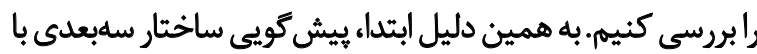

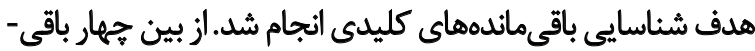

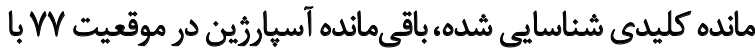

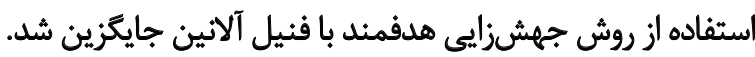

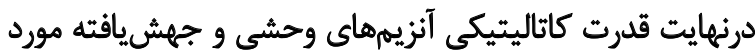

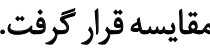
مواد و روشها

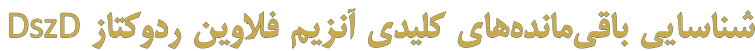

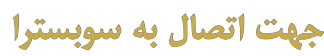

توالى آمينو اسيدهاى آنزيم DszD در بايكاه NCBI با عدد

1. IGTS8 Rhodococcus erythropolis 
شامل دى آمينوبنزيدين ه/ • ميلى زرم در ميلىليتر و هراكسيد

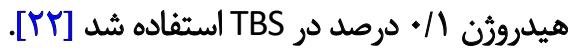

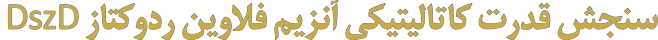

جهت بررسى قدرت كاتاليتيكى آنزيم DszD، ابتدا يروتئينهاى

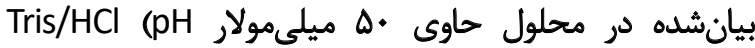

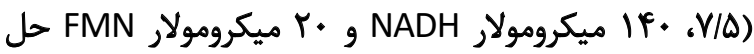

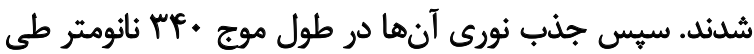

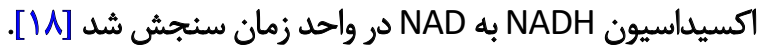

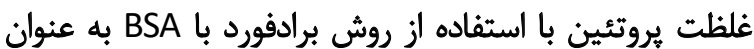

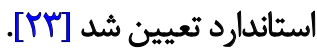

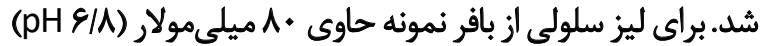
Tris-Hcl و معرف برموفنول آبى / • درصد استفاده شد برد.

جهت مشاهده الكوى يروتئينى،نمونههابر روى رُليلى آكريلاميد

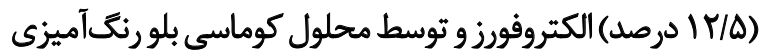

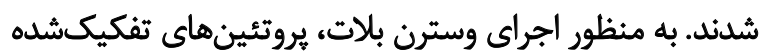

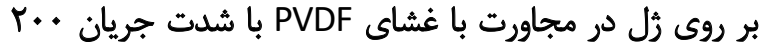

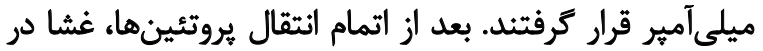

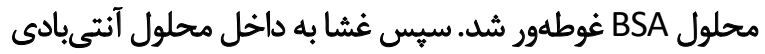

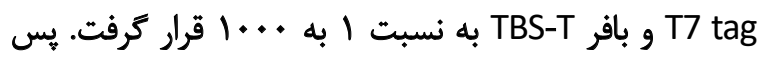

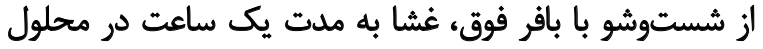
حاوى آنتىبادى ضد

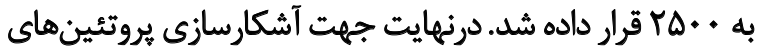

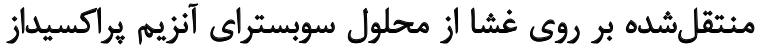

\section{GT\&M $\quad \forall Y V Q$}

1USF YPGVPAVVGVRVEERVNFCPAVWNTGLSADPPLFGVSISPKRFTHGLLLKARRFSASFHP 3ZOE YPGVPAVVGVRVEERVNFCPAVWNTGLSADP PLFGVSISPKRFTHGLLLKARRFSASFHP 3PFT PSGVIAIAAEV-DGTRVGLAASTFVPVSLEPPLVAFAVQNSSTTWPKLKDLPSLGISVLG DSZD PAGVTVVTLDS-GTGPVGFTATSFSSVSLEPPLVSFNIAETSSSINALKAAESLVIHLLG ЗСB0 AGAVQIVTTAG-AAGRRGLTLTAACSVSDNPPTILICLQKIHEENRIFIENGVFAINTLA $3 \mathrm{~K} 88$ 2D37

$4 \mathrm{XJ} 2$ STPVTVVATNG-PFGLAGLTCSAVCSVCDRPPTVLLCINRKSYAAGI IKSNGVLSVNWLA PLGVAIVTTNW-KGELVGMTVNTFNSLSLNPPLVSFFADRMKGNDIPYKESKYFVVNFTD ATGVTVVTTAG-PQGPLGMTVNSFSSVSLEPPLVLWCPARTSARHAAFAEAGAWSVHVLG ATGVTVVTASDAAGKPIGFTANSFTSVSLDPPLLLVCLAKSSRNYESMTSAGRFAINVLS TASSLTSVSLKPPLILFCLNKNSFS INSFQKSDKFAVS ILAENQIDISKHFAKSQPNKFT

$$
\text { تصوير ا. نتايج همترازى توالى آمينو اسيدهاى آنزيم فلاوين ردوكتاز DzSD با آنزيهماى همولوك }
$$

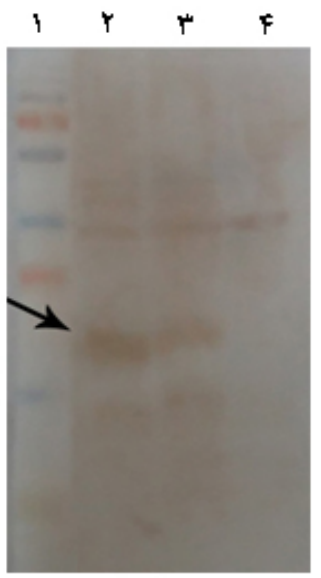

(飞)

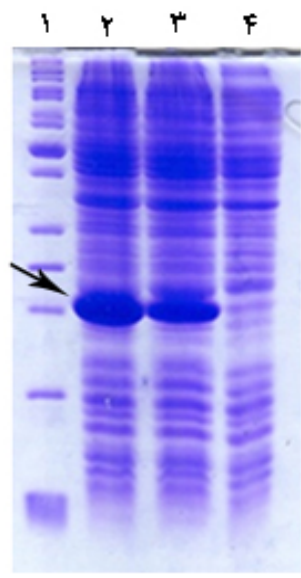

(ب)

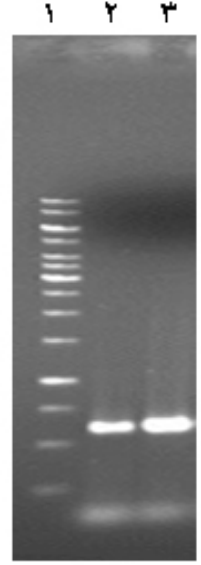

(الف)

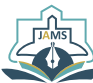

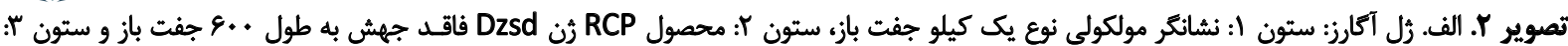

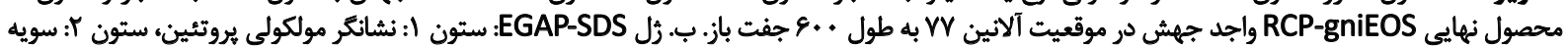

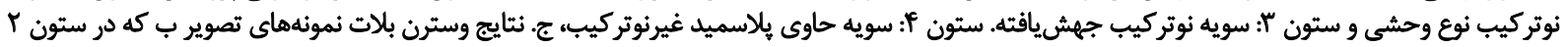

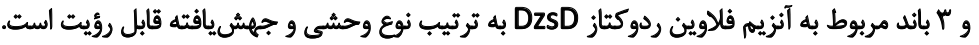


استفاده از روشهاى مهندسى رنتيك جهت حذف اختصاصى

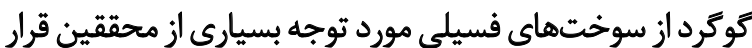

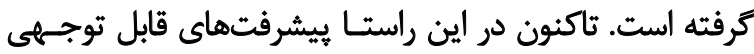

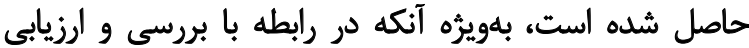

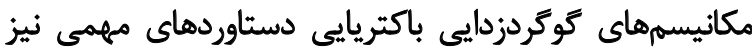

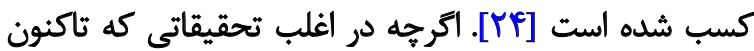

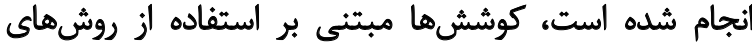

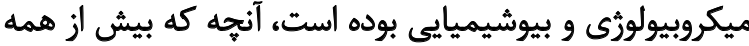

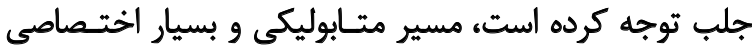

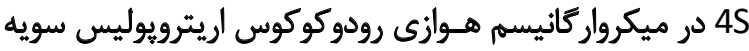

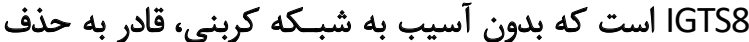

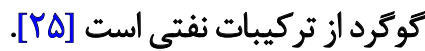

در فرايند 4S، آنزيم فلاوين ردوكتاز DszD به سهرم خود يك

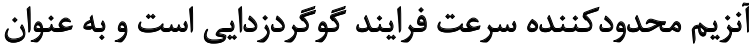

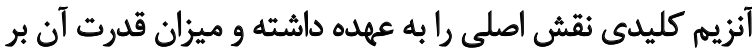

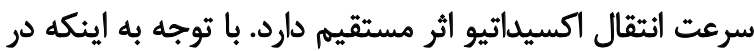

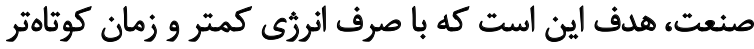

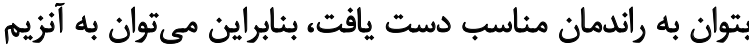

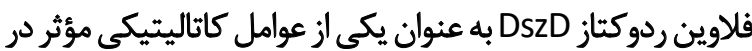

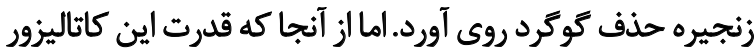

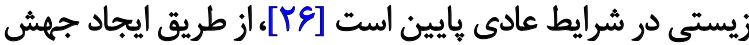

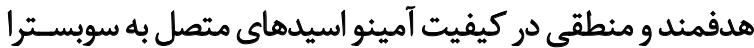

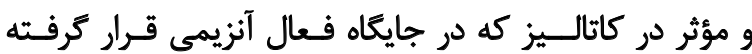

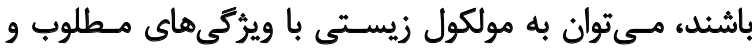

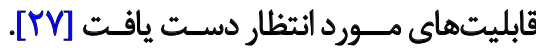

جن و همكاران مولكول جهشيافته جديدى از آنزيم ايزوينتيل

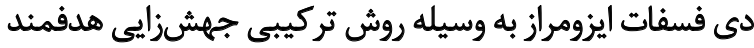

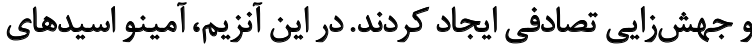

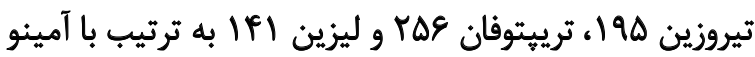

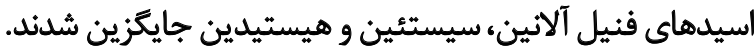

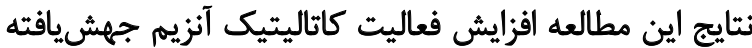

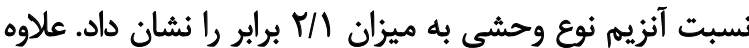

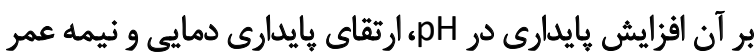

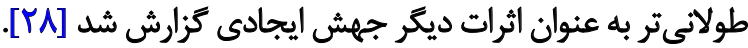
دوان و همكاران نيز مولكولهاى موتانت متعددى از آنزيم

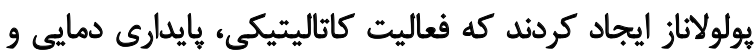

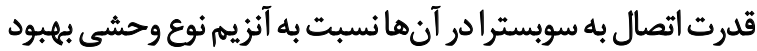

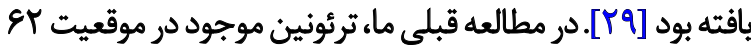

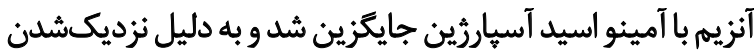

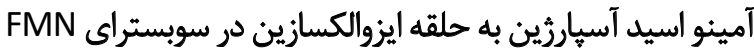

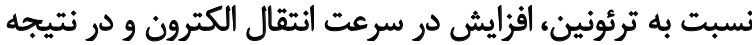

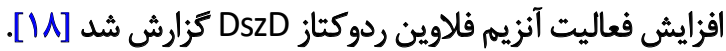
در اين مطالعه، فعاليت آنزيمى بعد از ايجاد جهش هدفمند
يافتهها

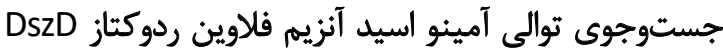

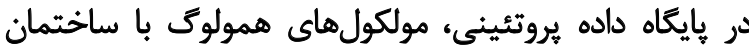

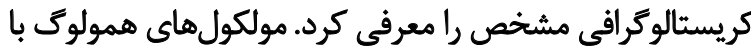

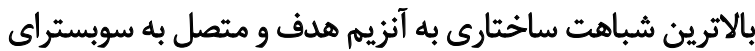

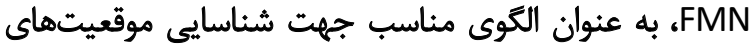

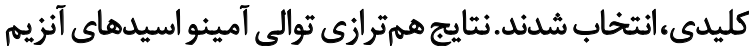

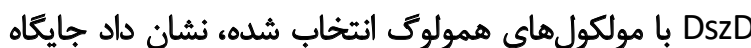

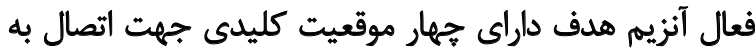

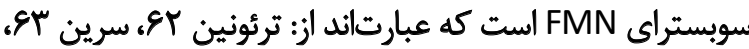

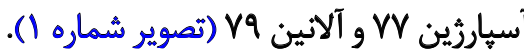

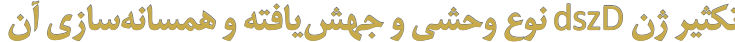

تكثير ثن dszD نوع وحشى با استفاده از يرايمرهاى اختصاصى (تون

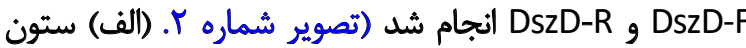

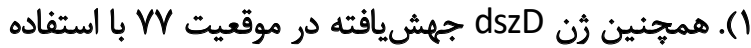

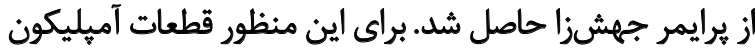

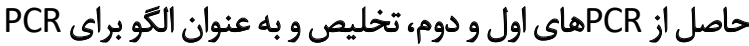

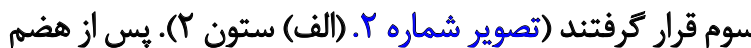

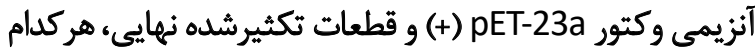

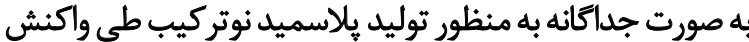

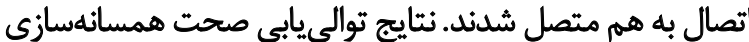

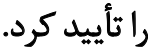

هيلاسميدهاى نوتركيب حاوى ثن نوع وحشى و جهشيافته

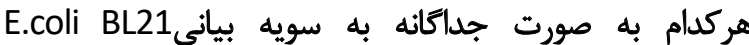

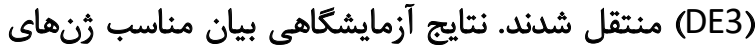

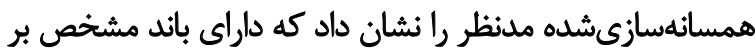

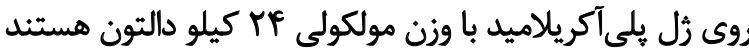

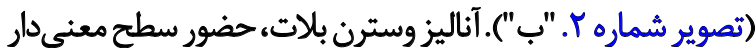

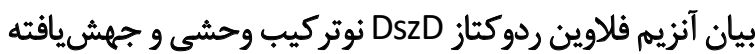

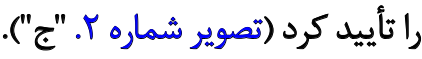

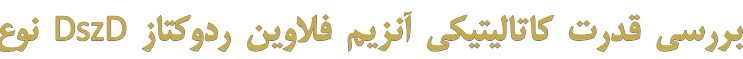

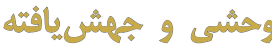

نتايج مقايسه ميانگين قدرت كاتاليتيكى آنزيم فلاوين ردوكتاز

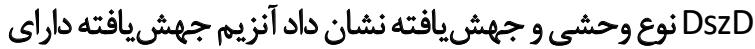

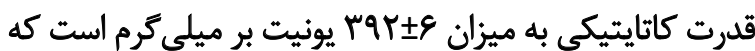

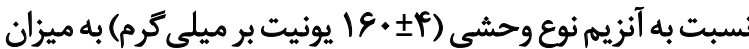

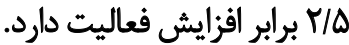
بحث امروزه به دليل تصويب قوانين سخت كيرانه زيستمحيطى، 


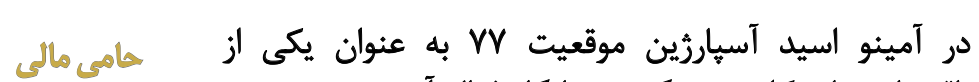

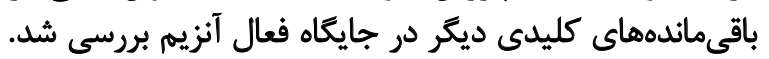
اين مطالعه بدون هركونه حمايت مالى صورت كرفته است.

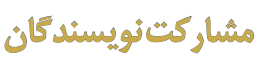

مفهومسازى، تحقيق و بررسى،ويراستارى ونهايىسازى نوشته: كليهنويسندكان.

$$
\text { تُعارض منأقع }
$$

نويسندكان تصريح مى كنيد هيجيُونه تضاد منافعى در اين

يثروهش وجود ندارد.

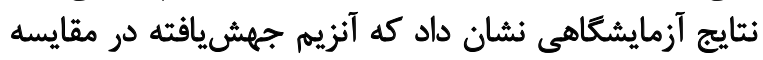

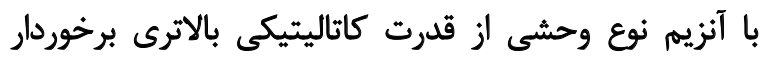

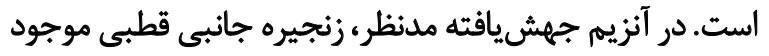

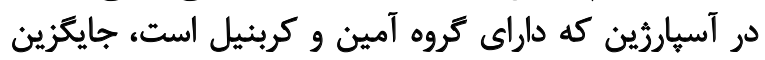

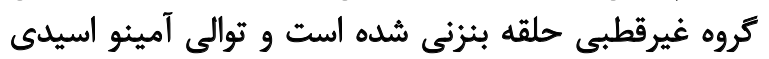

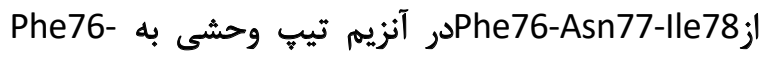
Phe77-Ile78 در آنزيم جهشيافته تغيير يافته است.

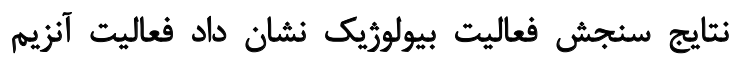

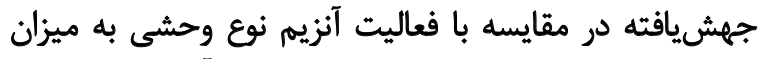

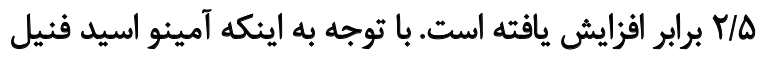

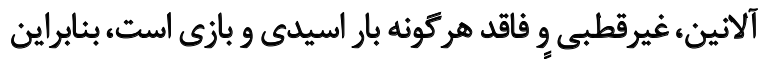

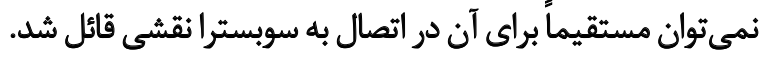

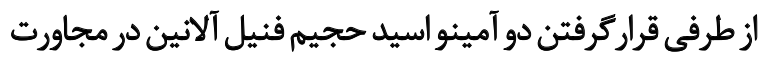

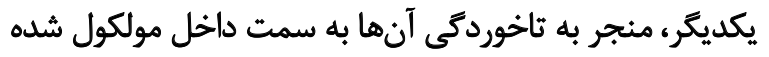

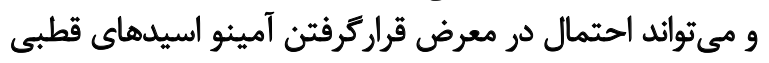

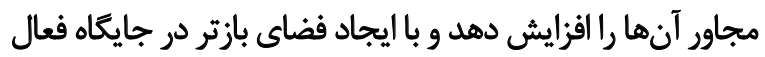

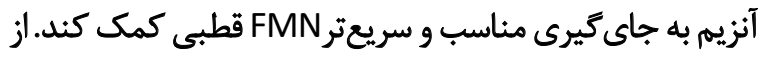

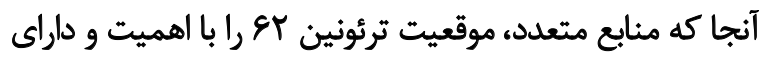

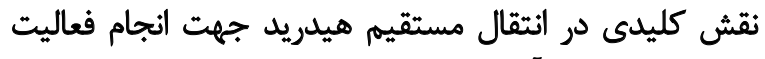

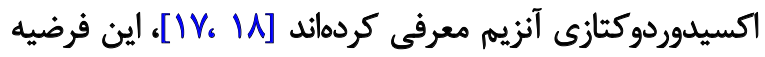

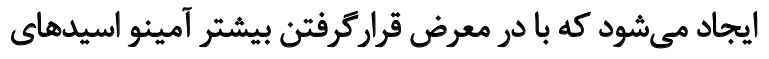

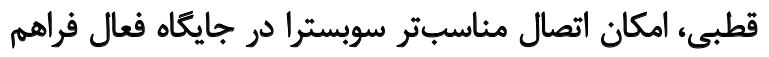
شده و درنتيجه فعاليت اكسيدوردوكتازى آنزيم افزايش مي ائيد

\section{تثيجلميرى}

اين مطالعه نشان مىدهد كه آنزيم مدنظر، بـانتانسيل مناسبى جهت افزايش قدرت كاتاليتيكى در مسير 4 دارئ دارد. بنابراين،

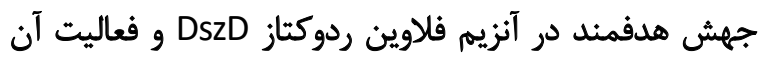

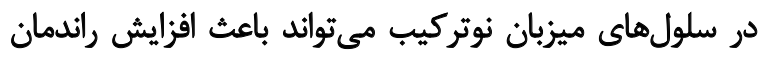

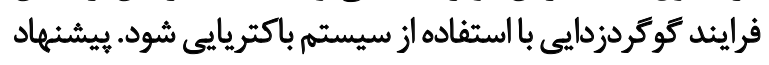

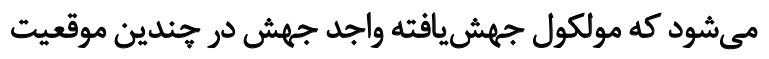

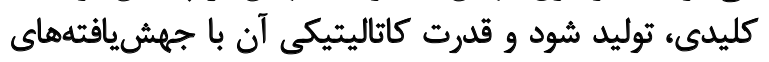
كزارش شده، مقايسه شود.

مالاحظات اخلاقى

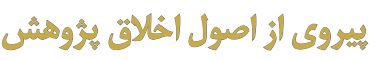

اين مطالعه با كد IR.NIGEB.EC.1398.6.24 A به تأييد

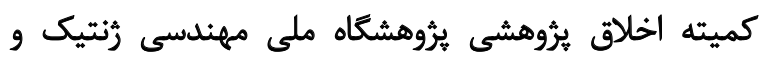

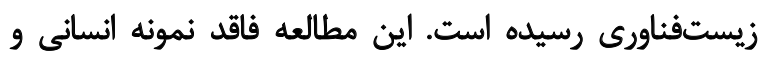

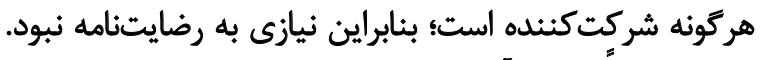
مطالعه صرفاً بر روى آنزيم صنعتى انجام شده است. 


\section{References}

[1] Etemadi N, Sepahy AA, Mohebali G, Yazdian F, Omidi M. Enhancement of bio-desulfurization capability of a newly isolated thermophilic bacterium using starch/iron nanoparticles in a controlled system. Int J biol macromol. 2018; 120:1801-9. [DOI:10.1016/j.jjbiomac.2018.09.110] [PMID]

[2] Kilbane II JJ. Microbial biocatalyst developments to upgrade fossil fuels. Curr Opin Biotechnol. 2006; 17(3):305-14. [DOI:10.1016/j.copbio.2006.04.005] [PMID]

[3] Chen S, Zhao C, Liu Q, Zang M, Liu C, Zhang Y. Thermophilic biodesulfurization and its application in oil desulfurization. Appl Microbiol Biotechnol. 2018; 102(21):9089-103. [DOI:10.1007/s00253-018-9342-5] [PMID]

[4] Nuhu AA. Bio-catalytic desulfurization of fossil fuels: A mini review. Rev in Envir Sci Bio/Technol. 2013; 12(1):9-23. [DOI:10.1007/s11157-0129267-x]

[5] Morrison E, Kantz A, Gassner GT, Sazinsky MH. Structure and mechanism of styrene monooxygenase reductase: New insight into the FADtransfer reaction. Biochem. 2013; 52(35):6063-75. [DOI:10.1021/ bi400763h] [PMID] [PMCID]

[6] Gupta N, Roychoudhury P, Deb J. Biotechnology of desulfurization of diesel: Prospects and challenges. Appl Microbiol Biotechnol. 2005; 66(4):356-66. [DOI:10.1007/s00253-004-1755-7] [PMID]

[7] Davoodi-Dehaghani F, Vosoughi M, Ziaee AA. Biodesulfurization of dibenzothiophene by a newly isolated Rhodococcus erythropolis strain. Bioresour Technol. 2010; 101(3):1102-5. [DOI:10.1016/j.biortech.2009.08.058] [PMID]

[8] Zhang M, Zhu W, Xun S, Li H, Gu Q, Zhao Z, et al. Deep oxidative desulfurization of dibenzothiophene with POM-based hybrid materials in ionic liquids. Chem engineer J. 2013; 220:328-36. [DOI:10.1016/j. cej.2012.11.138]

[9] Xiao J, Wang X, Fujii M, Yang Q, Song C. A novel approach for ultradeep adsorptive desulfurization of diesel fuel over $\mathrm{TiO}_{2}-\mathrm{CeO}_{2} / \mathrm{MCM}-48$ under ambient conditions. AIChE J. 2013; 59(5):1441-5. [DOI:10.1002/ aic.14085]

[10] Borzenkova N, Veselova I, Shekhovtsova T. Biochemical methods of crude hydrocarbon desulfurization. Biol Bull Rev. 2013; 3(4):296-311. [DOI:10.1134/S2079086413040026]

[11] Sucharitakul J, Tinikul R, Chaiyen P. Mechanisms of reduced flavin transfer in the two-component flavin-dependent monooxygenases. Arch biochem biophysics. 2014; 555-556:33-46. [DOI:10.1016/j. abb.2014.05.009] [PMID]

[12] Nazari F, Kefayati M, Raheb J. The study of biological technologies for the removal of sulfur compounds. J Sci, Islam Repub Iran. 2017; 28(3):205-19

[13] Karimi E, Yazdian F, Rasekh B, Jeffryes C, Rashedi H, Akhavan Sepah A, et al. DBT desulfurization by decorating bacteria using modified carbon nanotube. Fuel. 2018; 216:787-95. [DOI:10.1016/j.fuel.2017.10.030]

[14] Khosravinia S, Mahdavi MA, Gheshlaghi R, Dehghani H, Rasekh B. Construction and characterization of a new recombinant vector to remove sulfate repression of dsz promoter transcription in biodesulfurization of dibenzothiophene. Frontiers Microbiol. 2018; 9(1578):1-9. [DOI:10.3389/fmicb.2018.01578] [PMID] [PMCID]

[15] Martínez I, Mohamed ME-S, Santos VE, García JL, García-Ochoa F, Díaz E. Metabolic and process engineering for biodesulfuriza- tion in Gram-negative bacteria. J biotechnol. 2017; 262:47-55. [DOI:10.1016/j.jbiotec.2017.09.004] [PMID]

[16] Sousa SrF, Sousa JF, Barbosa AC, Ferreira CE, Neves RP, Ribeiro AJ, et al. Improving the biodesulfurization of crude oil and derivatives: A QM/MM investigation of the catalytic mechanism of NADH-FMN oxidoreductase (DszD). J Physical Chem. 2016; 120(27):5300-6. [DOI:10.1021/acs.jpca.6b01536] [PMID]

[17] Ferreira P, Sousa SF, Fernandes PA, Ramos MJ. Improving the catalytic power of the DszD enzyme for the biodesulfurization of crude oil and derivatives. Chem Eur J. 2017; 23(68):17231-41. [DOI:10.1002/ chem.201786864] [PMID]

[18] Kamali N, Tavallaie M, Bambai B, Karkhane AA, Miri M. Site-directed mutagenesis enhances the activity of NADH-FMN oxidoreductase (DszD) activity of Rhodococcus erythropolis. Biotechnol Letters. 2010; 32(7):921-7. [DOI:10.1007/s10529-010-0254-4] [PMID]

[19] Magrane M, Consortium U. UniProt knowledgebase: A hub of integrated protein data. Nat Prec. 2010. [DOI:10.1038/npre.2010.5092]

[20] Young L, Smith HO, Gibson DG. In vitro recombination method. San Diego: Google Patents; 2017.

[21] Sambrook J, Fritsch EF, Maniatis T. Molecular cloning: A laboratory manual. New York: Cold Spring Harbor Laboratory Press; 1989.

[22] Schägger H. Tricine-sds-page. Nat Protoc. 2006; 1(1):16-22. [DOI:10.1038/nprot.2006.4] [PMID]

[23] Kruger NJ. The Bradford method for protein quantitation. The protein protocols handbook. Berlin: Springer; 2002. [DOI:10.1385/159259-169-8:15]

[24] Wang J, Butler III RR, Wu F, Pombert JF, Kilbane II JJ, Stark BC. Enhancement of microbial biodesulfurization via genetic engineering and adaptive evolution. PloS One. 2017; 12(1):e0168833. [DOI:10.1371/journal.pone.0168833] [PMID] [PMCID]

[25] Akhtar N, Ghauri MA, Akhtar K. Dibenzothiophene desulfurization capability and evolutionary divergence of newly isolated bacteria. Arch Microbiol. 2016; 198(6):509-19. [DOI:10.1007/s00203-0161209-5] [PMID]

[26] Rangra S, Kabra M, Gupta V, Srivastava P. Improved conversion of Dibenzothiophene into sulfone by surface display of Dibenzothiophene monooxygenase (DszC) in recombinant Escherichia coli. J Biotechnol. 2018; 287:59-67. [DOI:10.1016/j.jbiotec.2018.10.004] [PMID]

[27] Vollhardt KPC, Schore NE. Organic chemistry; Palgrave version: Structure and function: Macmillan international higher education. New York: W H Freeman; 2014. [DOI:10.1007/978-1-319-191979_2]

[28] Chen H, Li M, Liu C, Zhang H, Xian M, Liu H. Enhancement of the catalytic activity of Isopentenyl Diphosphate Isomerase (IDI) from Saccharomyces cerevisiae through random and site-directed mutagenesis. Microbial Cell Factories. 2018; 17(1):1-14. [DOI:10.1186/ s12934-018-0913-z] [PMID] [PMCID]

[29] Duan X, Chen J, Wu J. Improving the thermostability and catalytic efficiency of Bacillus deramificans pullulanase by site-directed mutagenesis. Appl Environ Microbiol. 2013; 79(13):4072-7. [DOI:10.1128/AEM.00457-13] [PMID] [PMCID] 
This Page Intentionally Left Blank 